Bull. Mater. Sci., Vol. 9, No. 1, March 1987, pp. 67-72. C) Printed in India.

\title{
Nonlinearity in gold-alkali halide-gold capacitors
}

\author{
S C K MISRA \\ Department of Electrical Engineering, University of Strathclyde, Glasgow, UK \\ Present address: National Physical Laboratory, New Delhi 110 012, India \\ MS received 17 October 1986
}

\begin{abstract}
All epitaxial gold- $\mathrm{NaCl} / \mathrm{NaBr}$-gold capacitors were fabricated on hot $\mathrm{NaCl}$ substrates. The dielectric properties, capacitance and loss have been measured. Severe harmonic distortion in the output current has been observed. Maximum contribution was from third and odd harmonics. The amplitude of harmonics was maximum at frequencies above the loss peak. The phenomenon has been explained on the basis of nonlinear space charge theories.
\end{abstract}

Keywords. Gold; alkali halide gold; capacitors; nonlinearity.

\section{Introduction}

Thin film metal insulator devices have many advantages in integrated circuits and a large number of devices using this phenomenon have been made. Frequency modulation, switching, amplification, rectification and many more interesting phenomena are available from these devices. The practical device essentially comprises of metalsemiconductor-metal structure in one form or the other. The system is made either of bulk constituents or of thin films or hybrids. The large scale integration necessarily makes use of fabrication of devices by properly masking the base silicon chip and selectively preparing resistors, capacitors and inductors as in a FET. The thin film devices made from aluminium oxide, glasses, $\mathrm{CaF}, \mathrm{CdS}$, silicon oxide etc have been studied in great detail. For device fabrication, its properties have to be controlled for a successful operation. The electrical characteristics of the metal-electrode junction are nevertheless equally important. Invariably, charge injection or polarization is associated with a metal-semiconductor and metal-insulator structure. Polarization occurs mainly due to electronic blocking of charge carriers at the boundaries and electrode. The effects have been analyzed in thin films of such materials (Breckenridge 1950; Weaver 1962; Macfarlane and Weaver 1968).

Alkali halide thin films, because of their simplicity in structure, provide an ideal specimen for this purpose. The characteristics of the ionic charge carriers responsible for polarization are very well known. The nonlinear dielectric properties and mechanism of polarization in alkali halide thin films, both polycrystalline and epitaxial, have been studied. Various materials like $\mathrm{NaCl}, \mathrm{NaBr}$, Lif etc have also been studied. The dependence of dielectric constant and losses with the applied signal and by application of a d.c. bias have been analyzed quantitatively (Weaver 1975; Misra 1975). In all the above investigations epitaxial silver film was used as the electrode for the capacitor where the charge carriers are blocked. Since the phenomenon is a metal-insulator interfacial effect it was thought that the electrode material may have some effect on the dielectric response of metal-insulator-metal device, as it does in the semiconductor-metal devices. We have prepared capacitors having epi- 
taxial gold-alkali halide-gold structure. The dielectric properties of such devices are reported here.

\section{Experimental}

The gold-alkali halide $(\mathrm{NaCl})$-gold capacitor was fabricated on top of a hot $\mathrm{NaCl}$ crystal by a method similar to that used for silver electrode structures (Weaver 1975). The substrate temperature and the evaporation rate of gold were accordingly varied to get epitaxial gold film. Figure 1 shows the transmission electron micrograph of such a gold film (thickness $1000 \AA$ ) used as top electrode prepared at $250^{\circ} \mathrm{C}$ substrate temperature and $200 \mathrm{~V} / \mathrm{cm}$ electric field applied across the substrate during deposition. The absence of any polycrystallinity and the presence of stacking faults and dislocations are typical of epitaxial films.

Electrical measurements were carried out by continuously maintaining the specimen under vacuum. The method of measurement of dielectric loss and capacitance was the same as used earlier (Macfarlane and Weaver 1968; Weaver 1975).

\section{Results and discussion}

Figures 2 and 3 show the capacitance and dielectric loss for a $5000 \AA$ thick $\mathrm{NaCl}$ film capacitor with gold electrodes at temperatures $91^{\circ} \mathrm{C}, 118^{\circ} \mathrm{C}$ and $148^{\circ} \mathrm{C}$. It can be seen

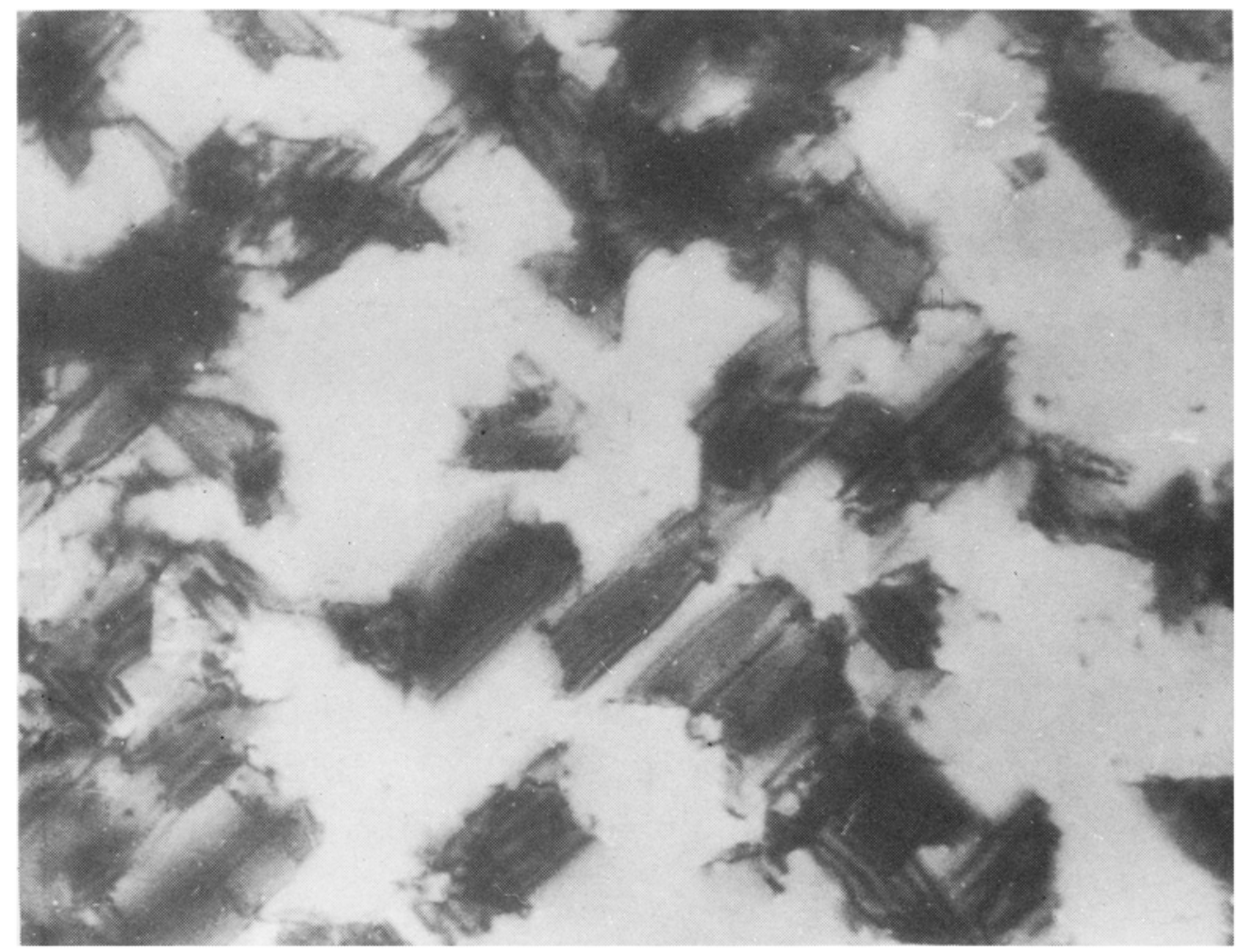

Figure 1. Electron micrograph of a $1000 \mathrm{~A}$ thick gold film $(\times 50,000)$. 


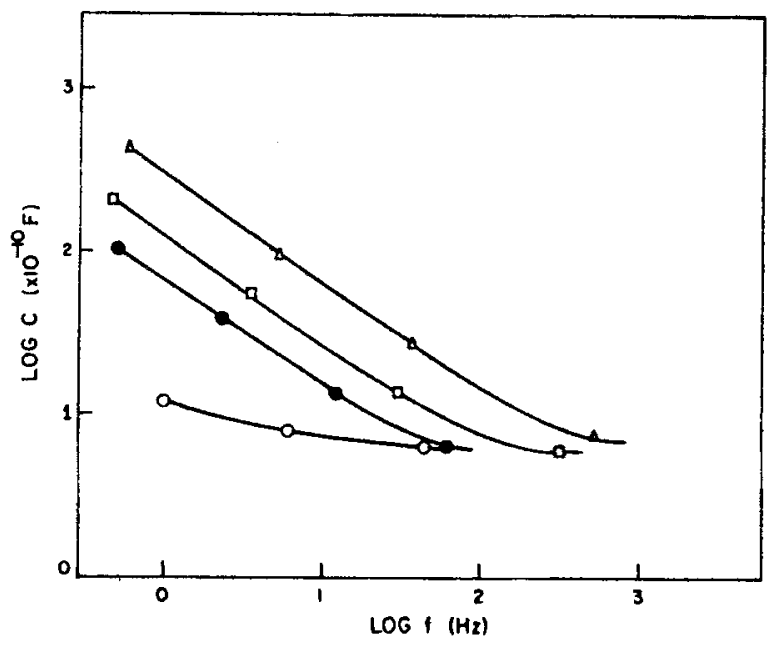

Figure 2. Variation in capacitance of epitaxial gold-NaCl-gold capacitor at $91^{\circ} \mathrm{C}$, $\square 118^{\circ} \mathrm{C}, \triangle 148^{\circ} \mathrm{C}, \bigcirc$ room temperature.

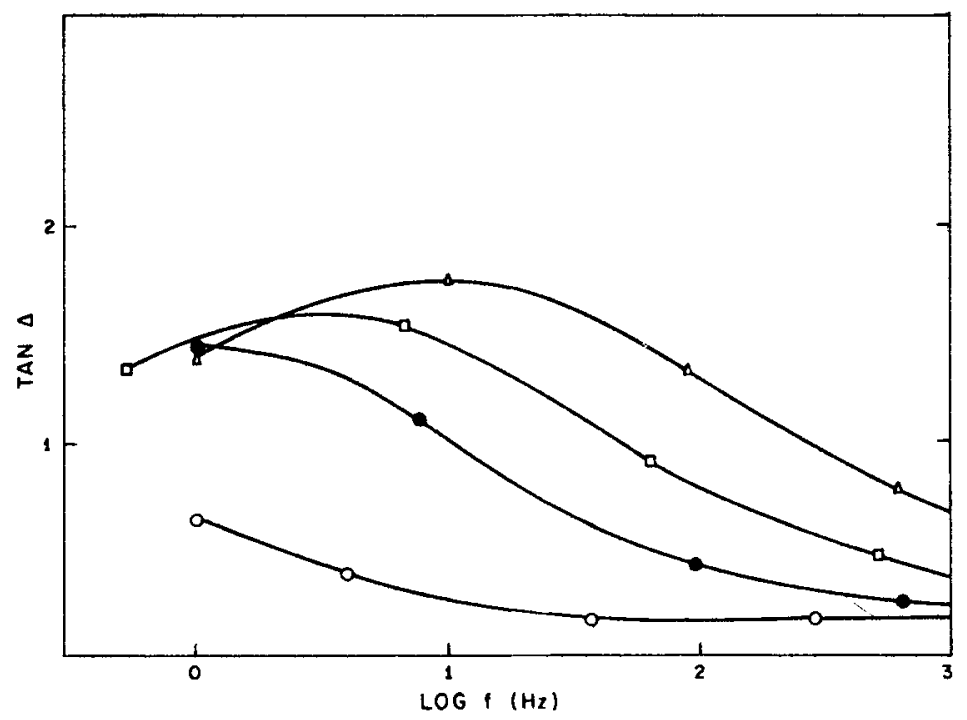

Figure 3. Variation in dielectric losses of epitaxial gold- $\mathrm{NaCl}$-gold capacitor at $91^{\circ} \mathrm{C}$, $\square 118^{\circ} \mathrm{C}, \triangle 148^{\circ} \mathrm{C}, \mathrm{O}$ room temperature.

that the loss peaks are broadened and shift to higher frequencies at higher temperatures. This can be attributed to the fact that the gold- $\mathrm{NaCl}$ interface may not be well defined, the gold film electrode deposited on $\mathrm{NaCl}$ film is not perfectly smooth and that the interface boundary is diffused.

However the presence of only one peak signifies the complete blocking of charge carriers at the electrodes. Figure 4 shows the Arrhenius plot for activation energy for the loss peaks depicted in figure 3 . The activation energy has been deduced as $0.82 \mathrm{eV}$ which is typical of migration of cation vacancies in $\mathrm{NaCl}$ (Macfarlane and 


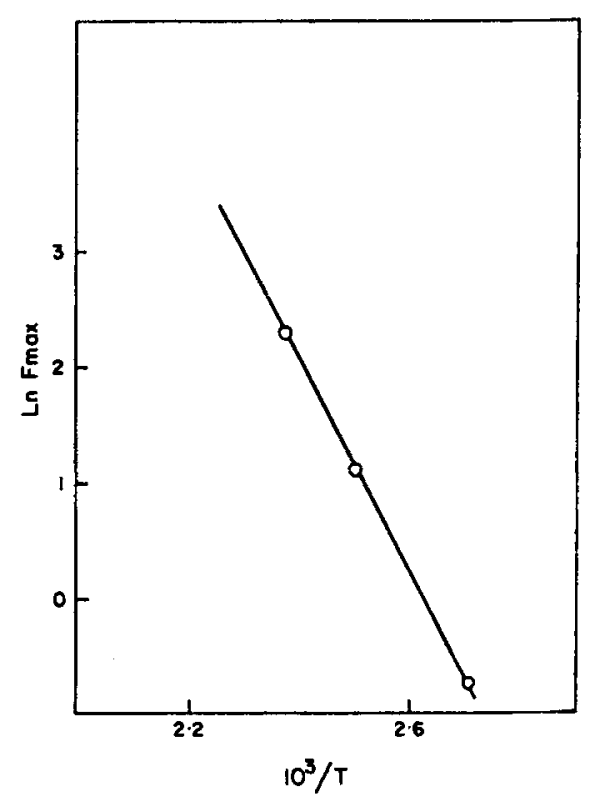

Figure 4. Arrhenius plot for activation energy.

Weaver 1968). This confirms that the only phenomenon taking place in our investigations is the migration of cation vacancies throughout the thickness of the film and thereupon complete blocking at the electrodes occur. The same conclusion was drawn while investigating the dielectric behaviour of $\mathrm{NaBr}$ films (Macfarlane and Weaver 1968$)$ where the activation energy $(1.05 \mathrm{eV})$ corresponding to that of migration of cation vacancies was obtained.

The capacitors exhibited a strong nonlinearity with the applied d.c. bias for any variation in the signal amplitude. The dielectric losses increased and distortion in the output waveform on application of a d.c. bias was observed. Variation in measuring the signal amplitude created a severe distortion of the output current waveform which contained distinct higher harmonics. We tried to measure the amplitude of harmonics present in the output current. A resonant filter was used in association with the phasemeter and the oscilloscope. Figure 5 shows the variation of amplitude of third harmonic of $5 \mathrm{~Hz}, 2 \mathrm{~Hz}$ and $20 \mathrm{~Hz}$ applied signal.

The loss peak for this specimen at $140^{\circ} \mathrm{C}$ was located around $5 \mathrm{~Hz}$. From figure 5 it can be seen that the amount of the third harmonic of input signal is maximum at $5 \mathrm{~Hz}$, which is the loss peak frequency. The harmonic content increased with increase in the signal amplitude.

It can be seen from figure 5 that modulation of $5 \mathrm{~Hz}$ signal to $15 \mathrm{~Hz}$ was maximum and increased tremendously with increase in the applied signal amplitude. For amplitudes in excess of $0.4 \mathrm{eV}$ where the field in the capacitor was of the order of $10^{6} \mathrm{~V} / \mathrm{M}$ the output rises sharply and then breaks down as seen on the oscilloscope. The highest frequency at which distortion occurs increased with temperature. No contribution from second and fourth harmonic of applied signal frequency could be detected. A very mild fifth harmonic was traceable which however could not be measured. 


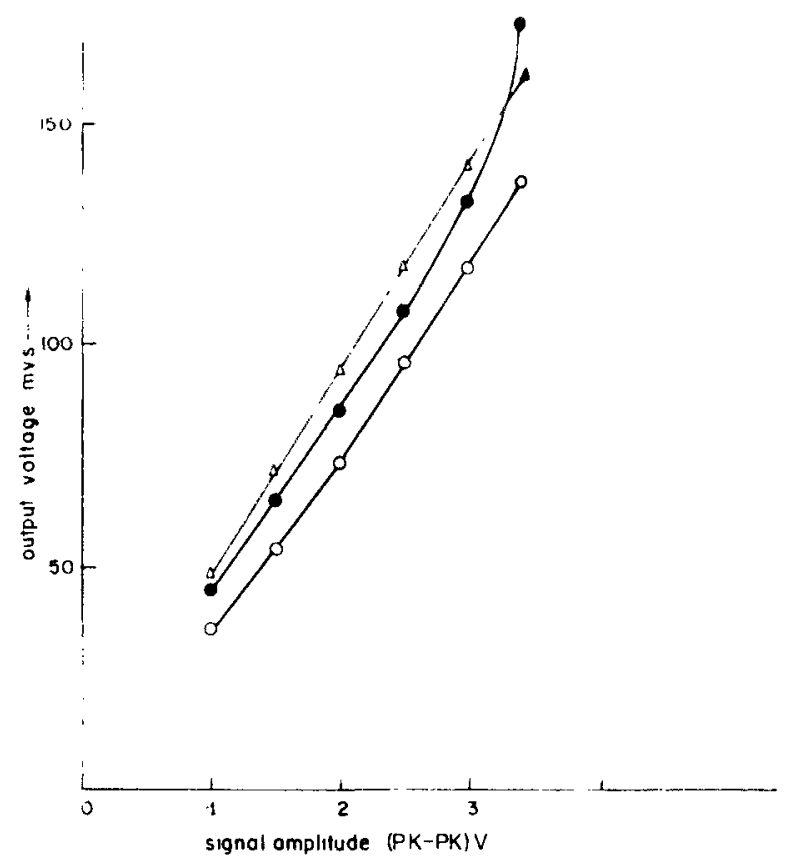

Figure 5. Vartation of the amplitude of third harmonic content with amplitude of input signal in a gold $\mathrm{NaCl}$-gold-capacitor at $118 \mathrm{C}$ for warious frequencies $-5 \mathrm{~Hz}, 2 \mathrm{~Hz}$, $20 \mathrm{~Hz}$.

To explain the linear dependence of dielectric loss and capacitance on the amplitude of the signal and distortion etc the simple theory of space charge polarization can be used. These theories require that the measuring signal should be less than $\mathrm{KT} / \mathrm{e}$, which means that it should be less than $10^{2} \mathrm{~V}$. For a $1000 \mathrm{~A}$ film we have used a signal in excess of $10^{2} \mathrm{~V}$ and therefore only nonlinear space charge theories can be applied. The present phenomenon was earlier predicted by the nonlinear space charge theory (Misra and Stern 1975). The application of an electric field across the capacitor creates a charge build up at the electrodes. The distribution of space charge gives rise to a field gradient. The gradient itself is field-dependent. In such a case the equations used to explain the polarization are highly complex and no longer linear. This eventually leads to nonlinearity of the dielectric behaviour. The current through the specimen will therefore contain higher and odd harmonics of the input signal. Such an effect has been observed in silicon oxide films (Argall and Jonscher 1968). This type of harmonic generation has been explained by Stern and Weaver. The harmonic generation should be observed only at or below the loss peak trequency and should contain contribution from third and higher odd harmonics. The same has been observed in the present investigations. Figure 6 shows the output current waveform for the specimen of figure 5.

\section{Conclusion}

The present investigations on metal alkali-halide metal structures also cover those with different electrode materials. Gold was used as the blocking electrodes. The 


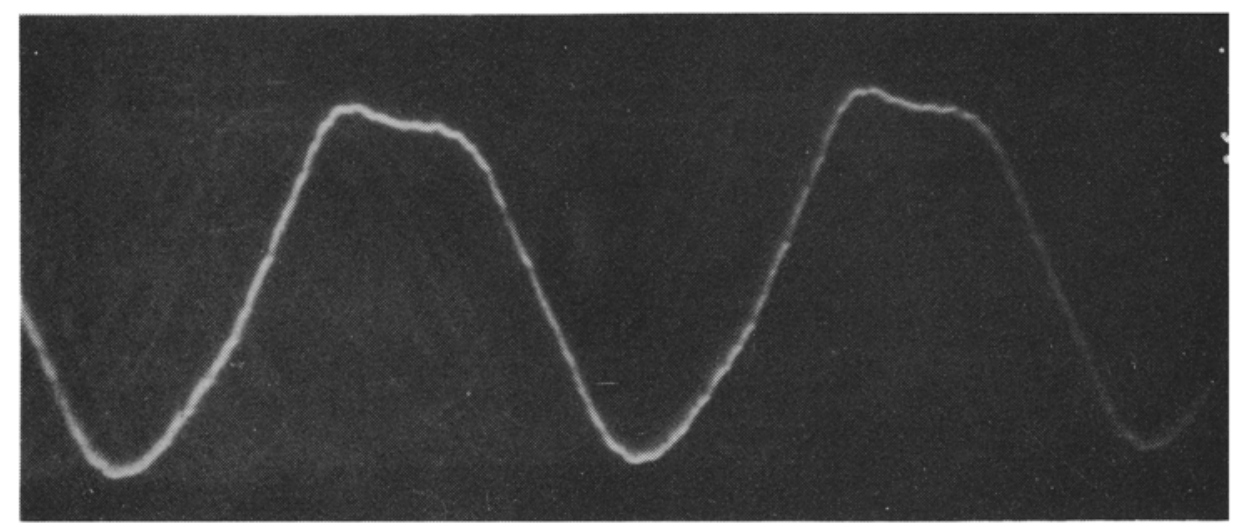

Figure 6. Output current waveform of the capacitor al $118 \mathrm{C}$. input signal $5 \mathrm{~Hz}$.

results show that replacing silver by gold does not in any case change the dielectric properties of thin film capacitor. The gold- $\mathrm{NaCl}$ interface is slightly different from silver- $\mathrm{NaCl}$ interface in that the loss peaks are not as sharp as in the latter and since the low frequency polarization in alkali halides is due to the migration of cation vacancies only, the electrode material has no effect.

The strong nonlinearity associated with space charge (interfacial) polarization leads to variation of dielectric constant and losses with applied signal. Frequency modulation of input signal below and around loss peak has been observed as is expected from nonlinear theories. The investigation of frequency modulation and accurate measurement of higher harmonics can be done. Similar phenomenon can be observed in complex structure materials like silicon oxide, aluminium oxide and cadmium sulphide etc. Once the phenomenon of frequency modulation is understood, the various parameters can be controlled and practical frequency modulation devices can be prepared from simple thin film capacitors.

\section{References}

Argall F and Jonscher A K 1968 Thin Solid Films 2185

Breckenridge R G $1950 \mathrm{~J}$. Chem. Phys. 16913

Macfarlane J C and Weaver C 1968 Philos. Mag. 1827

Misra S C K 1975 Ph.D. Thesis, Strathclyde University, UK

Misra S C K and Stern F 1975 Thin Solid Films 29339

Stern F and Weaver C 1970 J. Phys. C3 1736

Weaver C 1962 Adv. Phys. 283

Weaver C and Misra S C K 1975 Thin Solid Films 28303 